\title{
Reply to: Estrogens for protection from an index and recurrent episodes of takotsubo syndrome?
}

\section{Lu Fu',*, Hongyuan Zhang1,2,*, Jeremiah Ong'achwa Machuki', Tingting Zhang1,2, Lin Han', Lili Sang ${ }^{1}$, Lijuan Wu',2,

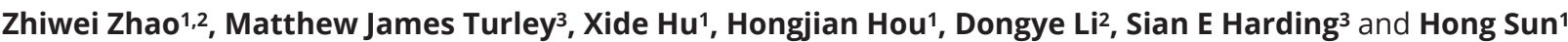

1Physiology Department, Xuzhou Medical University, Xuzhou, Jiangsu, China Institute of Cardiovascular Disease Research, Xuzhou Medical University, Xuzhou, China

${ }^{3}$ National Heart and Lung Institute, Imperial College London, London, UK

Correspondence should be addressed to S E Harding: shhli@ic.ac.uk

*(L Fu and $\mathrm{H}$ Zhang contributed equally to this work)

We appreciate the positive response to our paper 'GPER mediates estrogen cardioprotection against epinephrineinduced stress' from Prof Madias, published in this issue of Journal of Endocrinology pages L1-L2, and agree that it is important to understand whether a safe therapy can be designed to prevent takotsubo syndrome. This is particularly needed for previous patients, who have a higher risk of experiencing a recurrence. We do not have any published data on pre-treatment with a low dose of the GPER agonist G1 in our rat takotsubo model at present. The authors note that 'other therapies designed for ischemic coronary syndromes, including acute myocardial infarction have been recently proposed, and may need to be evaluated and tried in patients with TTS'. We have published data in a rat ovariectomy ex vivo cardiac ischemia/reperfusion model, using low dose estrogen sufficient to restore blood levels to around half of the pre-ovariectomy levels (Fu et al. 2017). This did not provide cardioprotection unless supplemented with low-dose testosterone.So, theaim offinding a dose of estrogen which will be cardioprotective without incurring its other problematic effects will be challenging. We agree that GPER agonists are a potential solution to that problem and consider it an excellent suggestion to titrate lower concentrations of G1 for pre-treatment in our rat TTS model.

Declaration of interest

The authors declare that there is no conflict of interest that could be perceived as prejudicing the impartiality of this article.

\section{Funding}

This work did not receive any specific grant from any funding agency in the public, commercial, or not-for-profit sector.

\section{References}

Fu L, Liu Y, Wang J, Sun Y, Zhang L, Wu Y, Li Y, Wang B, Huang S, Bu H, et al. 2017 Cardioprotection by low-dose of estrogen and testosterone at the physiological ratio on ovariectomized rats during ischemia/reperfusion injury. Journal of Cardiovascular Pharmacology 70 87-93. (https://doi. org/10.1097/FJC.0000000000000497)

Received in final form 18 June 2021

Accepted 19 July 2021 